\title{
The Influence of Disorder in Multifilament Yarns on the Bond Performance in Textile Reinforced Concrete
}

\begin{abstract}
M. Konrad, R. Chudoba
In this paper we analyze the performance of a bond layer between the multi-filament yarn and the cementitious matrix. The performance of the bond layer is a central issue in the development of textile-reinforced concrete. The changes in the microstructure during the loading result in distinguished failure mechanisms on the micro, meso and macro scales. The paper provides a brief review of these effects and describes a modeling strategy capable of reflecting the failure process. Using the model of the bond layer we illuminate the correspondence between the disorder in the microstructure of the yarn and the bonding behavior at the meso- and macro level. Particular interest is paid to the influence of irregularities in the micro-structure (relative differences in filament lengths, varying bond quality, bond-free length) for different levels of local bond quality between the filament surface and the matrix.
\end{abstract}

Keywords: Textile reinforced concrete, material model, bond performance, micro scale.

\section{Introduction}

Textile reinforced concrete (TRC) has emerged in the last decade as a new composite material combining the textile reinforcement with the cementitious matrix. Its appealing feature is the possibility to produce filigree high-performance structural elements that are not prone to corrosion, as is the case for steel reinforced concrete. In contrast to other composite materials, in TRC both the matrix and the reinforcement exhibit a high degree of heterogeneity of their material structure at similar scales of resolution. As a consequence, the fundamental failure mechanisms in the yarns, in the matrix and in the bond layer interact with each other and can result in several macroscopically different failure modes.

The development of a consistent material model for textile reinforced concrete requires the formulation and calibration of several sub-models on several scales of resolution. Each of these models represents the material structure at the corresponding scale (Fig. 1) with a focus on specific damage and failure mechanisms. The following correspondence between the scales and the observable components of the material structure and their interactions are specified:

- micro level

- filament, matrix

- bond filament - matrix

- meso level

$$
\begin{aligned}
& \text { - yarn, matrix } \\
& \text { - bond yarn - matrix }
\end{aligned}
$$

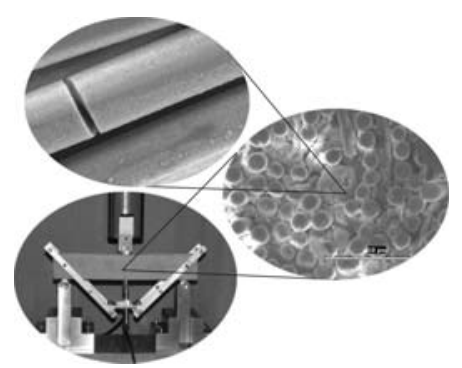

Fig. 1: Resolution scales
- macro level

$$
\begin{aligned}
& \text { - textile, matrix } \\
& \text { - bond textile - matrix }
\end{aligned}
$$

While models at the micro level are able to capture the fundamental failure and damage mechanisms of the material components (e.g. filament rupture and debonding from the matrix) their computational costs limit their application to small size representative unit cells of the material structure. On the other hand, macro level models provide sufficient performance at the expense of a limited range of applicability. Generally, all the scales must be included in the assessment of the material performance. The chain of models at each scale may be coupled (1) conceptually by clearly defining the correspondence between the material models at each level or (2) adaptively within a single multi-scale computation to balance accuracy and performance in an optimal way $[1,2]$.

Due to the complex structure of textile reinforced concrete at several levels (filament - yarn - textile - matrix) it is effective to develop a set of conceptually related sub-models for each structural level covering the selected phenomena of the material behavior. The homogenized effective material properties obtained at a lower level can be verified and validated using experiments and models at higher level(s).

The present paper is focused on the role of disorder in the bond layer between the yarn and the matrix. In Sec. 2 we review the elementary effects occurring in the bond layer during loading. After that, in Sec. 3, the model capturing some of these effects is introduced. Then, in Sec. 4, the calibration for a particular combination of yarn and matrix is performed, and finally, in Sec. 5, a parametric study shows the interaction effects between two failure mechanisms namely the debonding of filaments from the matrix and rupture of the filaments with an included disorder in the bundle.

\section{Elementary effects occurring in the bond layer}

In the reinforcement, the elementary mechanisms in the material behavior are appointed to the filaments with linear elastic behavior and brittle failure. The filament ensemble 
constituting the yarn exhibits nonlinear behavior due to disorder in the filament structure. The delayed activation of individual filaments leads to a gradual growth of stiffness at the beginning of the loading process, the friction between filaments influences the maximum stiffness reached during loading and both these effects influence the rate of failure after reaching the maximum force. Both in the filaments and in the yarn we may also observe the statistical size effect leading to reduced strength with increasing length [3].

The fine grained concrete matrix exhibits the evolution of microcracks in the fracture process zone that gradually close up to the macro crack. However, for the purpose of the present study, focused solely on the role of heterogeneity in the yarn, this influence can be disregarded.

The interaction between the reinforcement and the matrix can be seen on the micro- and meso-scales shown in Fig. 2. The fine scale interaction between the filament and the matrix includes the phases of bonding, debonding and friction. The interaction at the level of the yarn and matrix includes the same phases, but each of these phases includes fine scale interaction modes between the filaments and the matrix. Due to the complex structure of the failure process zone, the yarn-matrix bonding behavior cannot be captured without analyzing the interaction effects in micro-mechanical terms, as is done in this paper.

Another interaction effect occurs upon cracking of the matrix and the evolution of crack bridges leading to the tension stiffening effect in the overall response. This interaction is studied using meso-scale models, and goes beyond the scope of the present study [4]. The same holds for the interaction at the level of textile structures embedded in the matrix, which can be addressed by a macromechanical treatment $[5,6]$.

\section{Model of the bond layer}

In this model the interface layer between the yarn and the matrix is regarded as a set of laminas interacting with the matrix through the given bond law. The laminas represent groups of filaments with the same characteristics, and are coupled with the matrix using zero thickness interface elements [7].

The disorder in the filament bundle is taken into account using one of the three distributions of filament properties: (1) distribution of the bond quality, diminishing from the outside to the inside of the yarn (2) distribution of the bond free length, increasing from the outside to the inside of the yarn and (3) distribution of the delayed activation of filaments within the bond free length.

These distributions do not represent the disorder in the filament bundle directly. The bundle geometry is assumed in the form of a parallel set of filaments. The effect of disorder is reflected indirectly in terms of the mentioned distribution functions inducing an inhomogeneous stress transfer throughout the bond layer that is assumed to occur in a similar way in the heterogeneous material structure. The model can serve the purpose of capturing the influence of the variations in the bond performance on the macroscopically observable failure process, so that these variations may be quantified in a calibration procedure. The calibration of the model is performed using both the load-displacement curve and the curve representing the instantaneous fraction of the broken filaments during the loading process. The latter is obtained experimentally by optical recording of the light transmission through the unbroken filaments [8]. Using this model and the experimental data we are able to derive the effective bond law of the bond layer between the whole yarn

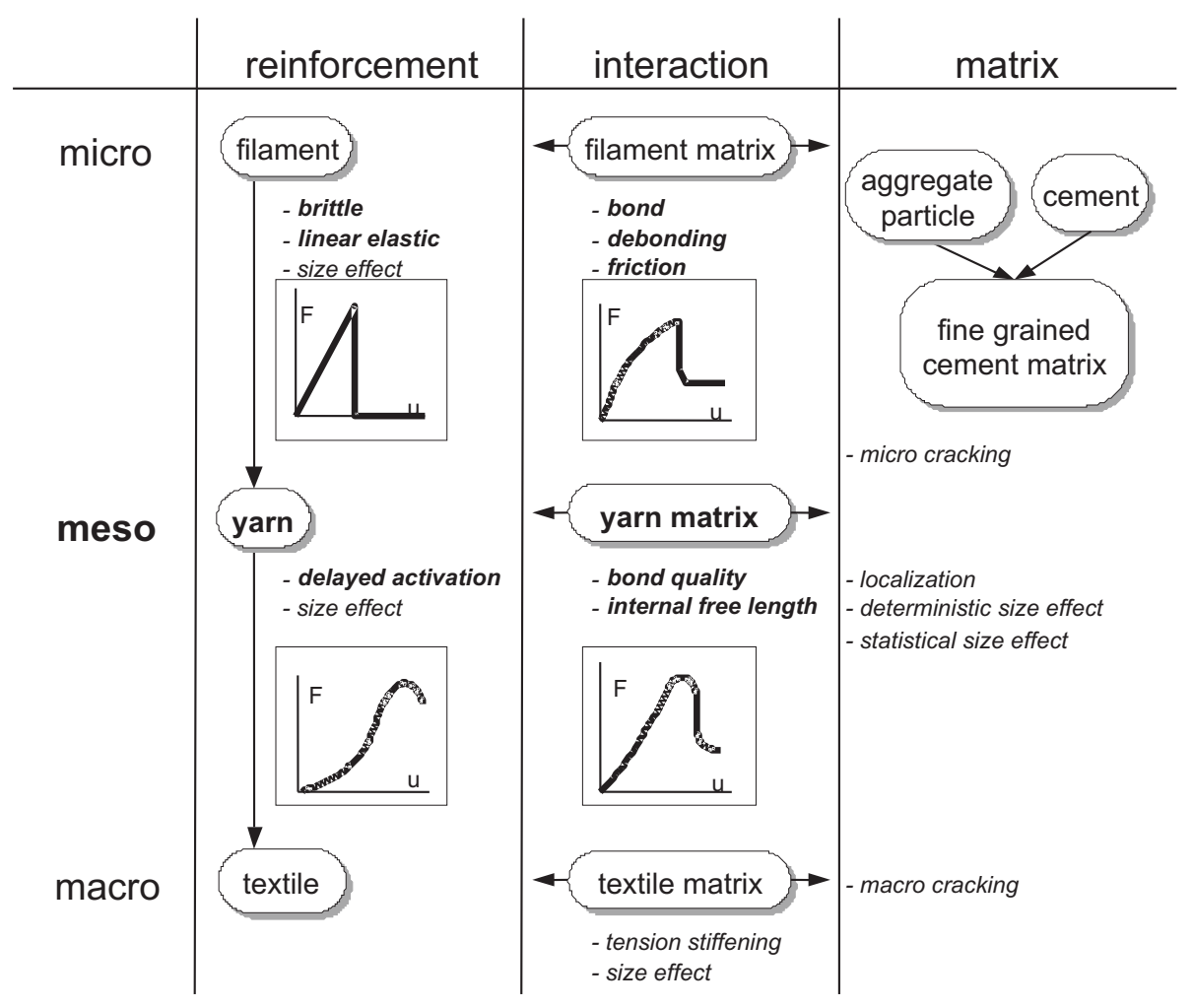

Fig. 2: Correspondence between scales, components and effects used in this model 
(filament bundle) and the matrix, which can be used at the higher modeling levels.

\section{Identification of the characteristic parameters for the bond layer}

For the selected combination of the yarn and the matrix, the parameters characterizing the tensile behavior of the yarn and the parameters of the bond between the filament surface and the matrix can be determined from the preliminary numerical and experimental study. In particular, the characteristics of the yarn and of the filaments can be derived from tensile tests on yarn. The applied stochastic modeling of the multi-filament bundle allowed us to obtain also statistical distributions of strength and stiffness along the yarn as described thoroughly in $[9,10]$. The local bonding between the filament surface and the matrix has been characterized by a bond model with parameters calibrated using the single filament pull-out experiment [11].

The sought material characteristics are the distributions of the bond quality, bond free length and activation strain across the filament bundle in the bond layer. The calibration procedure $[12,13]$ is based on the experimental data shown in Fig. 3. Here, the left diagram shows the load-displacement curves and the right diagram shows the diminishing fraction of unbroken filaments during the pull-out test for four selected specimens.

Before presenting the calibrated results, we first show the qualitative influence of the variations in the bond quality across the yarn cross section. Three examples of assumed bond quality distributions are shown in Fig. 4 with the maxi- mum achievable shear flow $(100 \%)$ at the outside of the yarn and linear, quadratic and cubic reduction in the internal layers.

The influence of a linear, a quadratic and a cubic bond quality distribution (Fig. 4) on the pull-out curve and on the progression of filament rupture is shown in Fig. 5. While the linear and the quadratic interpolation functions result in a sharp kink in the pull-out curve at the onset of filament rupture, the cubic distribution leads to a curve with a higher deformation capacity and is able to qualitatively reproduce the pull-out behavior and the progression of filament breaks measured in the experiment.

While the form of the bond quality distribution influences the post-peak slope of the pull-out curve the maximum pull-out force primarily depends on the tensile strength of the filaments. This correspondence is documented in Fig. 6 . Higher tensile strength of the filaments results in a higher pull-out force. Furthermore, higher filament strength leads to a higher frictional force at the end of the pull-out test because a greater number of filaments are pulled out prior to their rupture.

The effect of filament stiffness and strength and of the local bonding stiffness on the initial slope of the pull-out curve is not significant. On the other hand, their effect on the fraction of broken filaments is much higher. In other words, the initial stiffness in the pull-out test cannot be reproduced solely by reducing the bond quality and the tensile strength of the filaments. As a consequence, the reduced pull-out stiffness is explained by the existence of a free length inside the specimen between the macroscopic boundary of the matrix and the first contact of the filaments with the matrix inside the
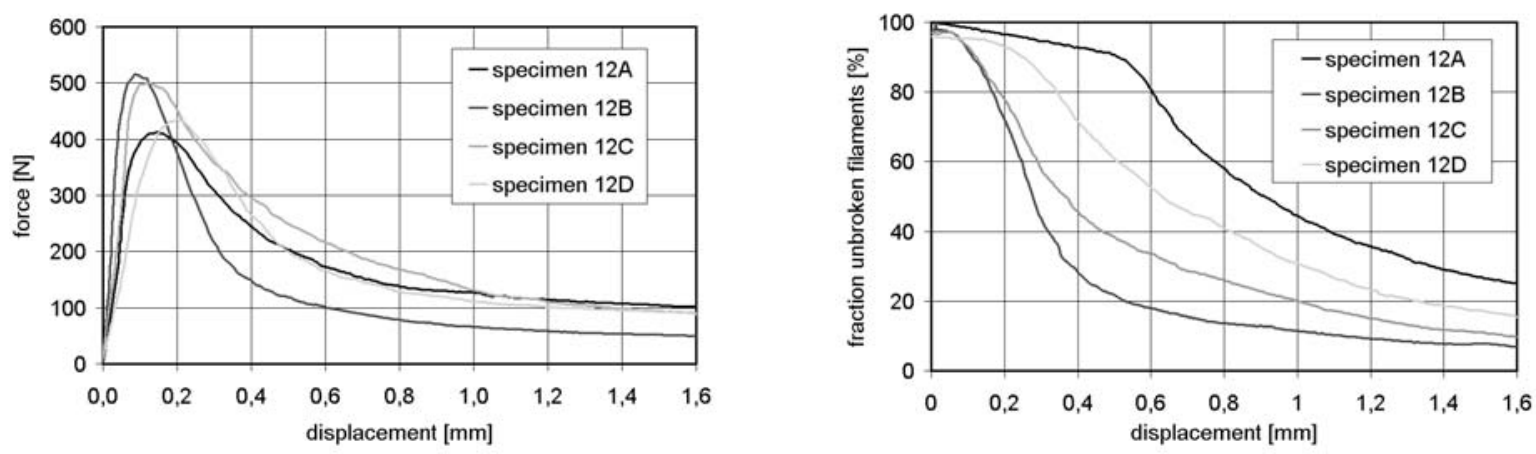

Fig. 3: Load-displacement curve, fraction of unbroken filaments
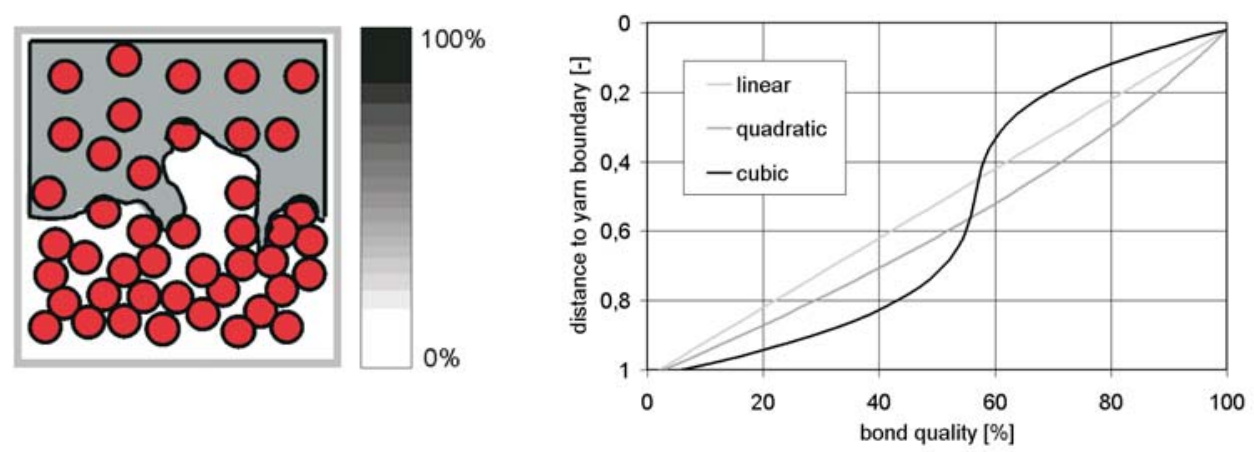

Fig. 4: Possible functions representing the decrease in the bond quality 

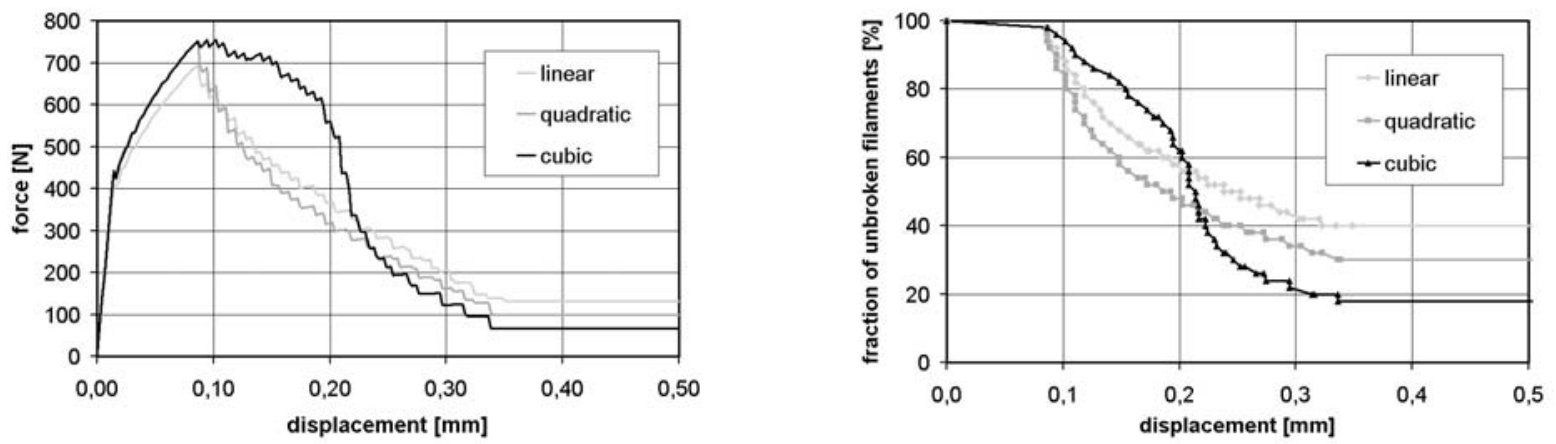

Fig. 5: Influence of different bond quality functions
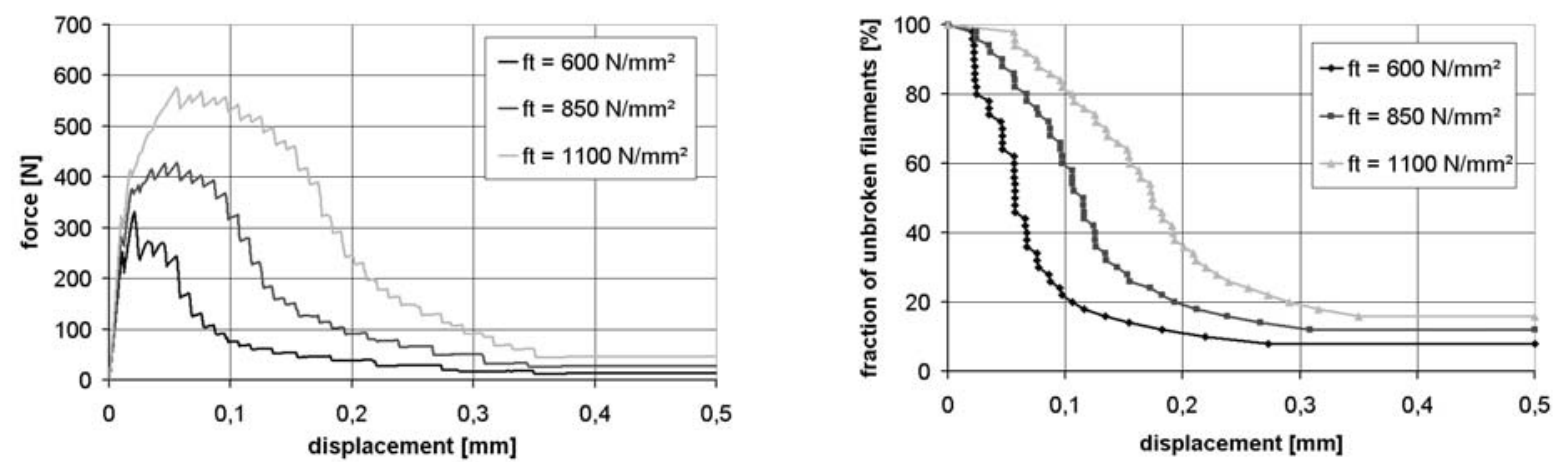

Fig. 6: Influence of filament strength $f_{t}$

specimen, i.e. the start of the microbonding between filament and matrix.

Similarly to the bond quality, we assume that the bond-free length increases from the outside of the yarn cross section to the inside, which is illustrated in Fig. 7. The influence of this free length on the initial stiffness is demonstrated in Fig. 8. The initial stiffness and the maximum pull-out force decrease with increasing bond-free length. Due to the shorter embedding length of the filaments the number of filaments being pulled out (i.e. debonded) increases and results in higher frictional force at the end of the pull-out test.

The filaments in the bundle exhibit a waviness which is illustrated in Fig. 9. Within the internal free length the filaments have the possibility to straighten before they get
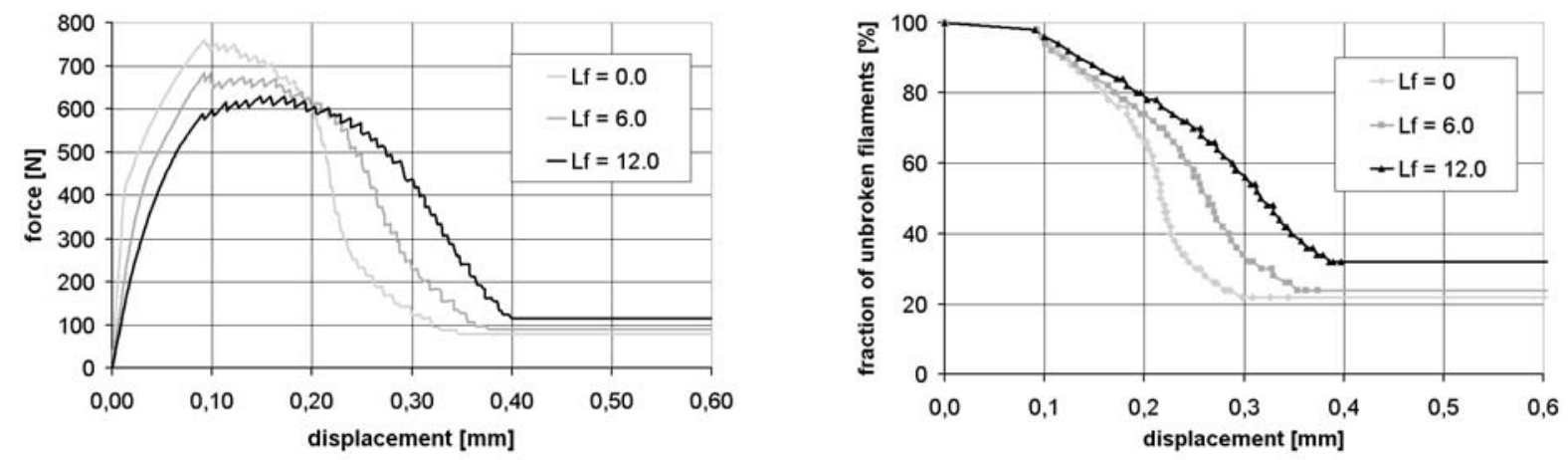

Fig. 8: Influence of free length 


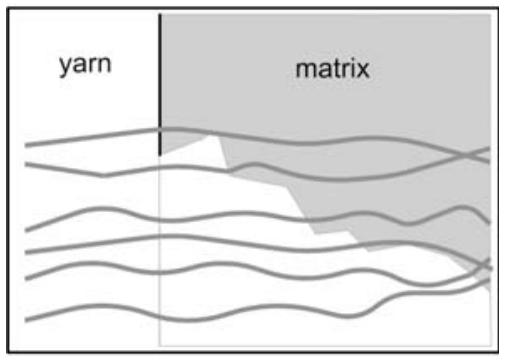

Fig. 9: Waviness of the filaments

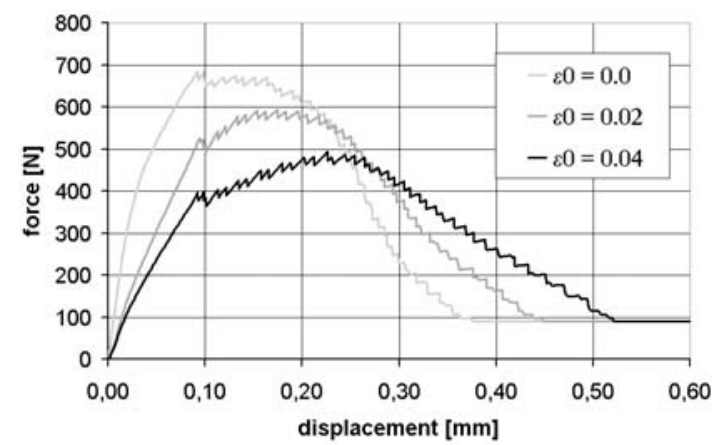

Fig. 10: Influence of activation strain

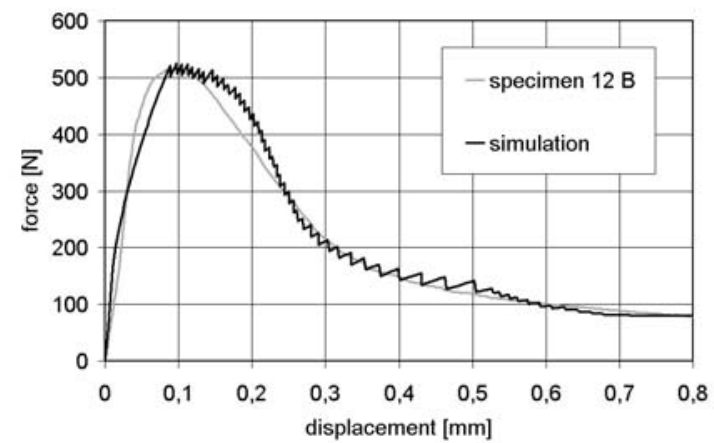

Fig. 11: Comparison of simulation and experiment

activated. The delayed activation of the individual filaments is modeled by an activation strain, which has to be reached before a filament takes up force. The activation strain increases with increasing free length. Fig. 10 shows the influence of lin-

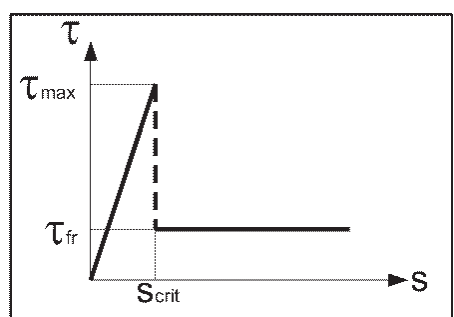

Fig. 12: Bilinear bond law ear distributions of the activation strain with different maxima is shown. The increasing delay of the activation results in further reduction of the initial stiffness and of the maximum pull-out force. In contrast to the parameters described above, it does not influence the number of broken filaments.

Using the parameters described above a calibration of the model is possible, as exemplified in Fig. 11. The calibrated distribution of the bond quality across the yarn cross section provides the basis for further modeling on the meso and macro level.
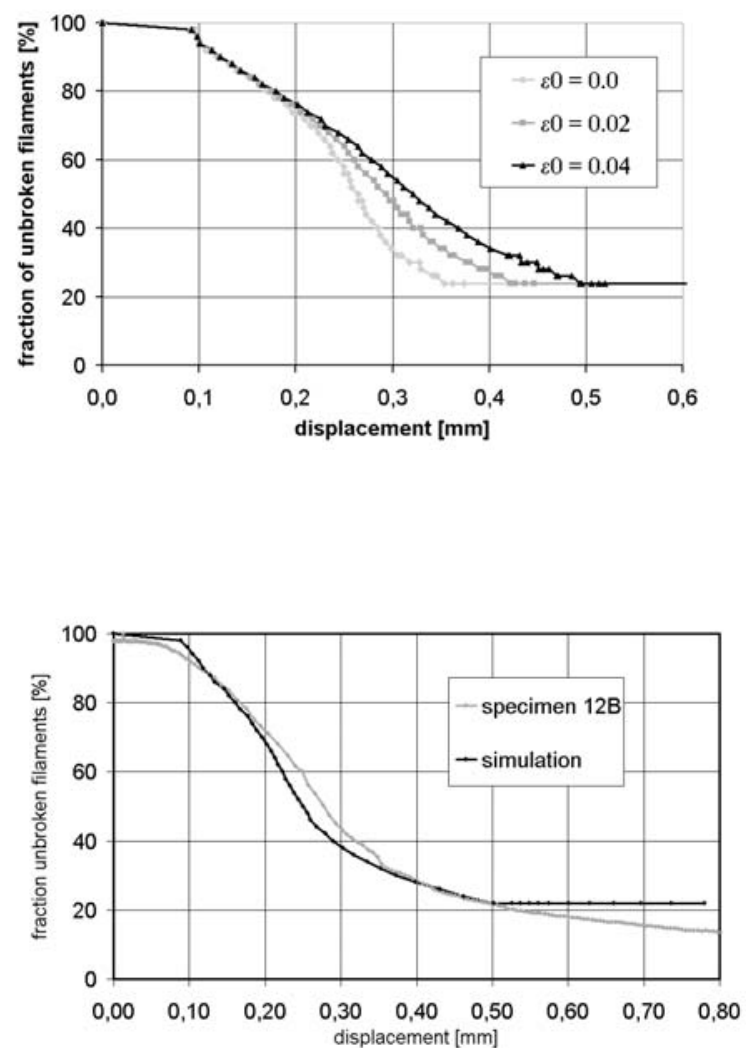

\section{Parametric study of the bond performance with disorder in the yarn}

In the previous section the characteristics of the material structure in the bond layer were introduced and their influence on the bond performance was shown. In the following we will study the influence of the local bond quality on the overall bond performance. As already specified, the bond behavior is described by a bilinear bond law (Fig. 12) including the phases of adhesive bond, debonding and friction. The local bond quality can be modified by changing the maximum bonding stress $\tau_{\max }$, the frictional stress $\tau_{\mathrm{fr}}$ and their ratio $\tau_{\max } / \tau_{\text {fr }}$

The influence of the maximum bond stress $\tau_{\max }$ and the ratio of maximum bond stress and frictional stress $\tau_{\max } / \tau_{\mathrm{fr}}$ 
for an embedding length of $30 \mathrm{~mm}$ is shown in Fig. 13. It is obvious that the effect of the maximum shear stress on the maximum pull-out force is negligible. This is a result of the long embedding length of $30 \mathrm{~mm}$ accumulating a high amount of the frictional stress. Therefore the maximum pull-out force is depends essentially only on the frictional stress.

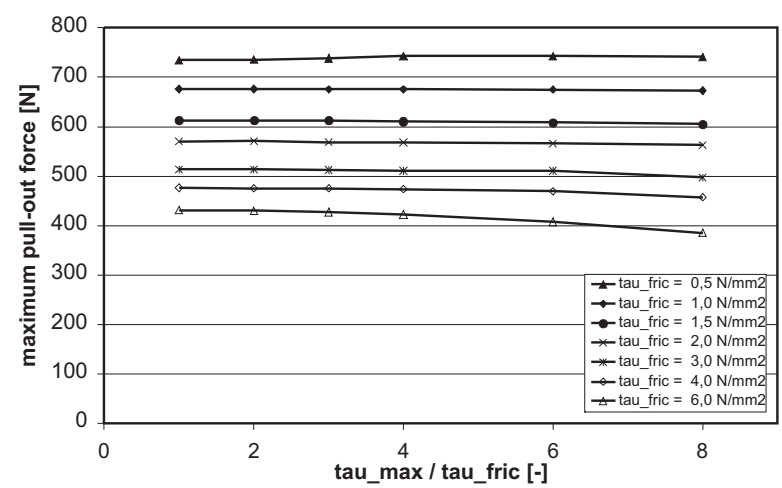

Fig. 13: Maximum pull-out force

The dependency of the maximum pull-out force on the frictional stress $\tau_{\mathrm{fr}}$ is shown in Fig. 14. For a constant maximum bond stress $\tau_{\max }$ the maximum pull-out force and the associated displacement decrease with increasing frictional stress tfr. This effect is rather surprising. It means that the improvement of the bond performance of the filament sur-

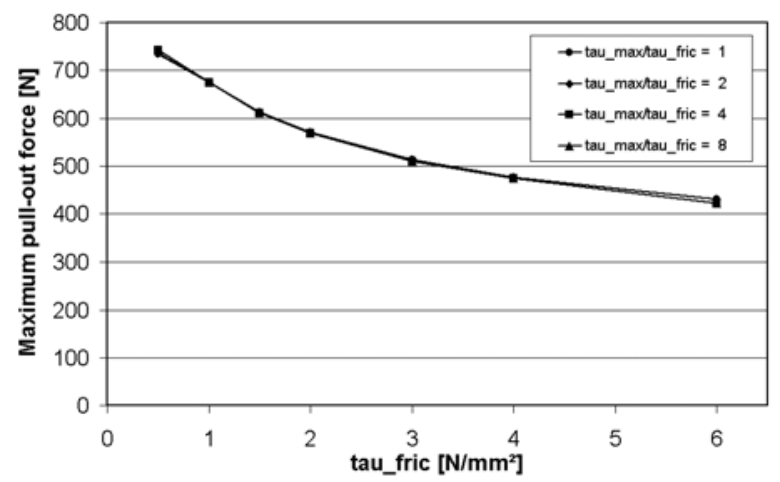

Fig. 14: Maximum pull-out force and associated displacement

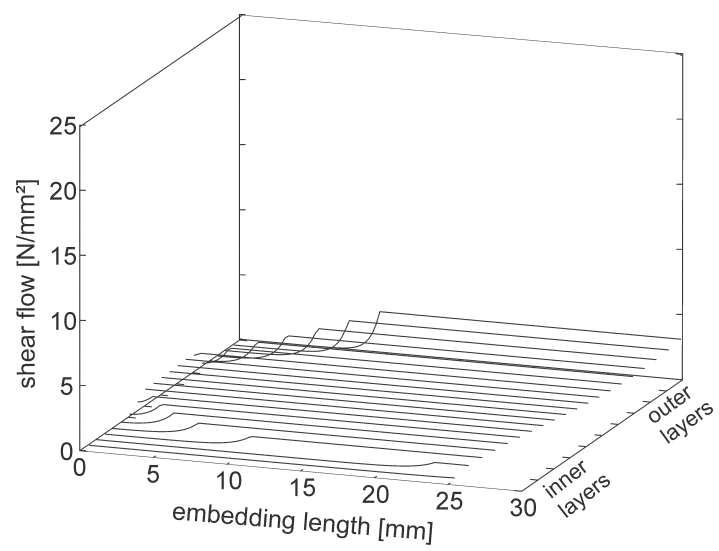

Fig. 15: Shear flow for $\tau_{\mathrm{fr}}=0.5 \mathrm{~N} / \mathrm{mm}^{2}$ and $\tau_{\mathrm{fr}}=6 \mathrm{~N} / \mathrm{mm}^{2}$ face results in a reduction of the resulting bond performance of the bundle.

In order to illuminate this effect, the shear flow at maximum pull-out force along the filament is displayed for each lamina in Fig. 15. The laminas in front represent the filaments inside the bundle with a low bond performance, while the rear laminas represent the outer filaments with a high bond performance. A constant shear flow indicates that up to this length the filaments have debonded. The left diagram shows the shear flow distribution across the bond layer for a low level of frictional stress $\tau_{\text {fr. }}$. The length activated for the stress transfer between the filaments and the matrix is much longer than in the right diagram, showing the shear flow distribution for a higher frictional stress. The longer stress transfer length results in a lower strain of the filaments, and leads to filament rupture at larger control displacements.

The reduction of the maximum pull-out force with increasing local bond strength is explained using Fig. 16. The two diagrams show the accumulative pull-out response (thick curve) and the pull-out curves for each lamina separately. For the lower level of frictional stress $\left(\tau_{\mathrm{fr}}=0.5 \mathrm{~N} / \mathrm{mm}^{2}\right)$ more filaments can be activated simultaneously (left diagram). At the maximum pull-out force there are $95 \%$ of the filaments active and at the end of the loading only $15 \%$ of the filaments get broken while all the rest remain intact.

On the other hand, for yarn with a higher level of friction $\left(\tau_{\mathrm{fr}}=6 \mathrm{~N} / \mathrm{mm}^{2}\right)$ there are only $55 \%$ of the filaments active at the maximum pull-out force (30\% are still inactive and $15 \%$
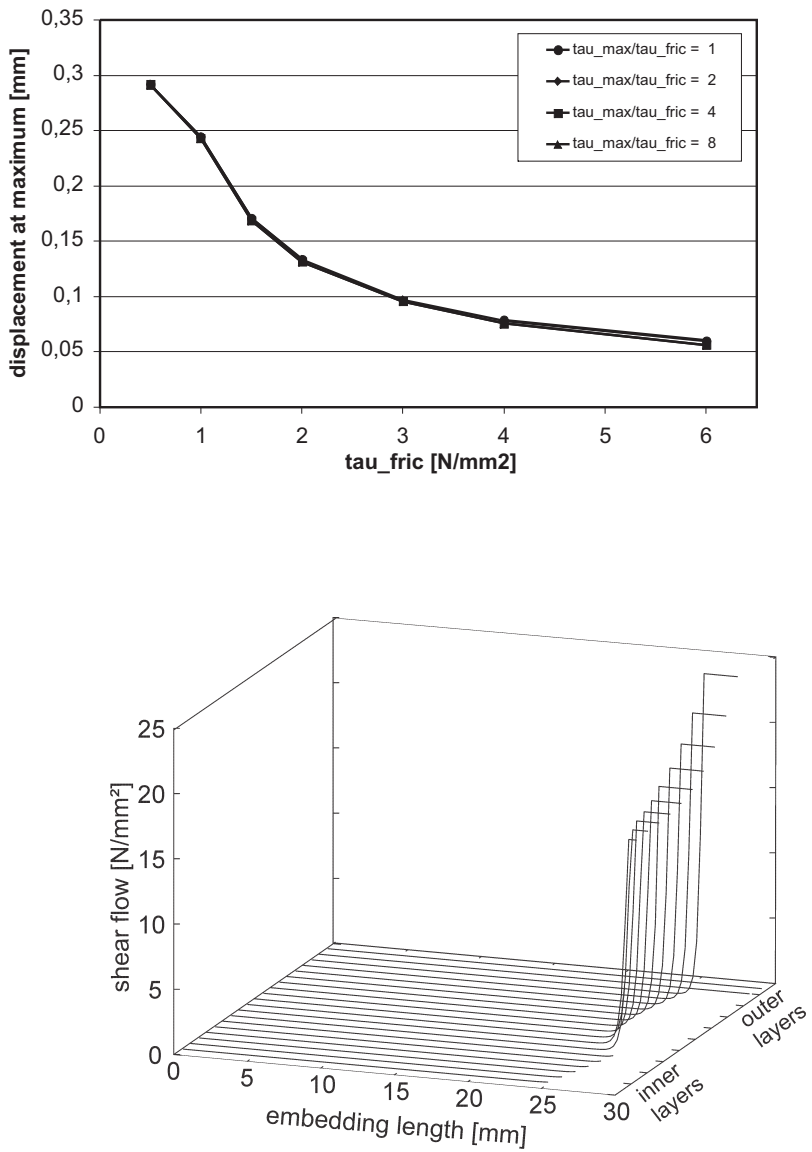


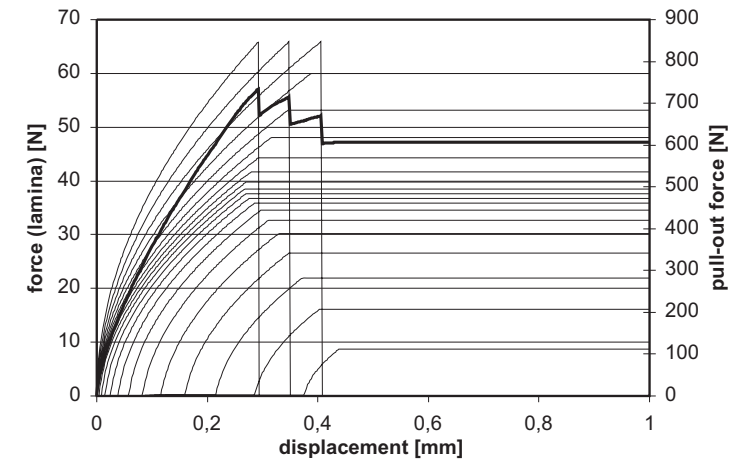

Fig. 16: Failure process for $\tau_{\mathrm{fr}}=0.5 \mathrm{~N} / \mathrm{mm}^{2}$ and $\tau_{\mathrm{fr}}=6 \mathrm{~N} / \mathrm{mm}^{2}$

are already broken). At the end of the loading all the filaments are broken. Thus, even though the inner filaments were able to transfer a higher amount of force to the matrix the resulting pull-out force was reduced, due to the non-uniformity of the transfer.

This qualitative comparison demonstrates the role of disorder represented by the varying bond-free length, delayed activation and spatial variations in the bond quality. The improvement of the local bond performance is counterproductive and results in an earlier failure of the outer filaments with higher bond performance. Due to the increased pull-out stiffness, the maximum pull-out force reaches its maximum at a smaller control displacement. At this displacement most of the inner filaments with lower bond performance have not been activated and cannot contribute to the total pull-out force.

\section{Conclusions}

In this paper, a modeling strategy for supporting the development of textile reinforced concrete is presented. It is based on the assumption that there is no ultimate model able to capture all the aspects of the material behavior. Therefore the models currently being developed in the framework of the collaborative research center are classified and evaluated with respect to the failure mechanisms being captured. It is important that they have a defined validity and clearly specified interfaces. They are applied together in order to study the material response at various scales of material resolution.

The modeling of the bond layer demonstrated that we face a failure process zone with complex interaction of elementary effects. The parametric study emphasized the role of disorder in this interaction and exemplified that it reverses the expected correlation between input parameters and material response.

The final message of this paper can be put as follows: In the design of cementitious composites reinforced with multi-filament yarns, the issue of disorder must be carefully analyzed. Only with a good knowledge of the phenomena in the microstructure it is possible to balance the performance of the individual components of the material structure to obtain optimum performance of the composite.

\section{Acknowledgment}

This work has been carried out in the framework of the project Simulation of Bond and Crack Behavior of Textile-Rein-

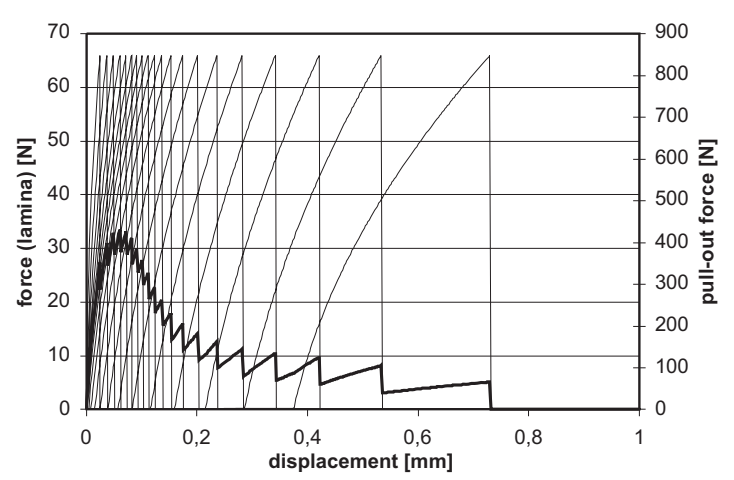

forced Concrete at the Meso Level included in the collaborative research center Textile Reinforced Concrete: Foundation of a New Technology (SFB 532) sponsored by the German Research Foundation.

\section{References}

[1] Krause R: "Investigation of failure mechanisms with a multiscale finite element method." Proc. European Conference on Computational Mechanics, München, 1999.

[2] Fish J., Yu Q.: "Computational mechanics of fatigue and life predictions for composite materials and structures." Computer Methods in Applied Mechanics and Engineering, Vol. 191 (2002), p. 4827-4849.

[3] Phoenix S. L.: "Stochastic strength and fatigue of fiber bundles." International Journal of Fracture, Vol. 14 (1978), No. 3, p. 327-344.

[4] Hegger J., Mombartz M., Chudoba R.: “Combined Use of Extended Finite Elements and H-Adaptivity for Simultaneous Crack Propagation and Strain Localization.” EUROMECH Colloquium 460, Numerical Modelling of Concrete Cracking, Innsbruck, Austria, 2005.

[5] Hegger J., Bruckermann O., Chudoba R.: "Modelling of the Bond of Filaments and Rovings." Proc., $2^{\text {nd }}$ Colloquium on Textile Reinforced Structures (CTRS2), Dresden, 2003.

[6] Hegger J., Bruckermann O., Voss S., Chudoba R.: “A Smeared Model for the Simulation of Textile Reinforced Concrete Tesion Tests." Proc., $3{ }^{\text {rd }}$ Asia-Pacific Conference on Fibre Reinforced Materials, Changsha, 2003.

[7] Kaliakin V. N., Li J.: "Insight into deficiencies associated with commonly used zero-thickness interface elements." Computers and Geotechnics, Vol. 17 (1995), No. 2 p. 225-252

[8] Brameshuber W., Banholzer B., Gries T., Al-Masri A.: "Methode zur Untersuchung des Versagensmechanismus unter Zugbelastung von Multifilament-Garnen für die Betonbewehrung." Technische Textilien, Vol. 45 (2002), p. 98-99.

[9] Chudoba R., Vorechovsky M., Konrad M.: "Stochastic modeling of multi-filament yarns I: Random properties within the cross-section and the size effect." Journal of Engineering Mechanics, submitted for publication, (2004). 
[10] Vorechovsky M., Chudoba R.: "Stochastic modeling of multi-filament yarns I: Random properties over the length and the size effect." Journal of Engineering Mechanics, submitted for publication, (2004).

[11] Brameshuber W., Banholzer B.: "Bond characteristics of filaments embedded in fine grained concrete.” Proc. $2^{\text {nd }}$ Colloquium on Textile Reinforced Structures (CTRS2), Dresden, 2003.

[12] Chudoba R., Butenweg C., Peiffer F.: "Textile reinforced concrete part I: Process model for collaborative research and development." Proc. International Conference on the Applications of Computer Science and Mathematics in Architecture and Civil Engineering, Weimar, 2003.
[13] Chudoba R., Pfeiffer F., Meskouris K.: "Experiment Design and Automated Evaluation under the Application of Numerical Material Models." Proc. $2^{\text {nd }}$ Colloquium on Textile Reinforced Structures (CTRS2), Dresden, 2003.

Ing. Martin Konrad, CSc.

Prof. Ing. Rostislav Chudoba, CSc.

e-mail: r.chudoba@aut.uni.de

Aachen University of Technology

Chair of Structural Statics and Dynamics, Mies-van-der-Rohe-Str 1

Aachen, Germany 